\title{
Guía
}

\section{Recomendaciones para la prevención de la transmisión perinatal del Virus de la Inmunodeficiencia Humana en Costa Rica}

\author{
(Recommendations for the prevention of perinatal transmission \\ of the Human Immunodeficiency Virus in Costa Rica)
}

\author{
Oscar Porras, Ricardo Boza, María-Paz León-Bratti, Alfredo Messino, Antonio Solano
}

Comisión Interhospitalaria de Terapia Antirretroviral, Caja Costarricense de Seguro Social, San José, Costa Rica Abreviaturas: $\mathrm{VIH}$, virus de inmunodeficiencia humana; TARV, terapia antirretroviral; $\mathrm{CVIHa}$, clínica $\mathrm{VIH} /$ Sida de adultos; CVIH-HNN, clínica de infección por VIH Hospital Nacional de Niños; AZT, Zidovudina; 3TC, Lamivudina; LPV, lopinavir/ritonavir; PR-VIH, prueba rápida para $\mathrm{VIH}$; PANI, Patronato Nacional de la Infancia.

Correspondencia: Oscar Porras, Servicio de Inmunología y Reumatología Pediátrica, Hospital Nacional de Niños "Dr. Carlos Sáenz Herrera", Apartado 1654, 1000 San José, Costa Rica. Teléfono 2223 5I 25. Email:porrasza@racsa.co.cr

ISSN 0001-6002/2008/50/4/243-247 Acta Médica Costarricense, (O2008 Colegio de Médicos y Cirujanos

\section{Resumen:}

Se conoce que la transmisión perinatal del VIH de madre a hijo es prevenible con la toma de algunas medidas generales y otras específicas. La acción fundamental para lograr esta prevención es identificar cuales embarazadas son portadoras del VIH temprano durante el embarazo. Para lograr este objetivo es necesario realizar la prueba del ELISA para VIH a toda mujer embarazada en su primera consulta prenatal. Las presentes son unas guías sobre el tema desarrolladas con el fin de facilitar la aplicación de todas las acciones necesarias para la prevención de la transmisión perinatal en Costa Rica, brindando una óptima atención médica a la madre y el recién nacido.

Descriptores: virus de la inmunodeficiencia humana, VIH, transmisión perinatal , embarazo, antirretrovirales, lactancia materna, BCG.

\section{Abstract:}

It is known that the perinatal transmission of HIV from mother to child is preventable applying some general and some specific measures. The basic action to achive the prevention is to identify which pregnant women are HIV positive early during the pregnancy. To obtain this aim it is fundamental to carry out the HIV-ELISA test to every pregnant woman early during the pregnancy. The present guides were developed to help aply all the preventive actions necessary to achive the prevention of perinatal HIV transmission in Costa Rica, offering an optimal medical attention to the mother and the newborn.

Keywords: human immunodeficiency virus, HIV, perinatal transmisión, pregnancy, antirretroviral, breastfeeding, BCG

La posibilidad de que una mujer embarazada y portadora de virus de la inmunodeficiencia humana (VIH) transmita el virus al producto es entre $25-45 \% .^{1}$ Desde la aparición de los resultados del protocolo ACTG 076 en 1994 donde se demostró que la profilaxis con Zidovudina (AZT) a las mujeres embarazadas y portadoras de VIH reducía la probabilidad de infección del producto hasta $67 \%,{ }^{2}$ el uso de terapia antirretroviral preventiva se ha generalizado. Estudios posteriores demostraron que estos resultados se podían obtener también en mujeres con enfermedad avanzada. Como se conoce que la mayor probabilidad de transmisión durante el embarazo es en el periodo perinatal, se estableció la pauta de realizar cesárea electiva a toda paciente portadora de VIH. ${ }^{3}$ Tanto el uso de AZT como la cesárea electiva son factores independientes pero sumatorios para la disminución de la transmisión perinatal del VIH. Con ambos se disminuyó la posibilidad de transmisión vertical a $2 \%$. 
En los últimos años se ha discutido la posibilidad de ampliar la profilaxis con AZT a terapia antirretroviral (TARV) triple a toda mujer embarazada portadora de VIH, basándose en la premisa de que la carga viral es un factor de riesgo para la transmisión vertical del VIH durante el embarazo. Se ha demostrado que el uso de triple terapia antirretroviral junto con la cesárea programada, disminuye a $1.2 \%$ la posibilidad de transmisión del virus. ${ }^{3,4}$ Sin embargo, debemos recordar que se han encontrado datos conflictivos, es decir la transmisión se ha reportado en madres con todos los niveles de carga viral, incluyendo no detectable.

Los antirretrovirales (ARV) muestran diferencias farmacocinéticas en mujeres embarazadas con respecto a otros grupos de pacientes. No se conocen los efectos secundarios a mediano y a largo plazo de estos medicamentos sobre los fetos y los niños. Durante el embarazo, algunos cambios en la tolerancia gastrointestinal de las mujeres hacen que sea difícil el uso de medicamentos y los ARV sabemos que producen diversos grados de efectos secundarios gastrointestinales.

Tomando en cuenta al binomio madre / producto, la decisión del uso de ARV debe ser discutida con la madre y es después del común acuerdo entre el equipo de atención interdisciplinario y la madre que se deberá escoger el esquema adecuado. ${ }^{5}$

Basados en la evidencia publicada, ${ }^{6-11}$ la Comisión Interhospitalaria de Tratamiento Antirretroviral de la Caja Costarricense de Seguro Social, recomienda el siguiente manejo para la prevención de la transmisión perinatal del $\mathrm{VIH}$ en la mujer embarazada infectada. Esta estrategia esta sustentada en tres elementos:

1. Tamizaje de la mujer embarazada en la primera consulta prenatal, idealmente durante el primer trimestre, pero

Cuadro 1. Actividades para la prevención de la transmisión perinatal del VIH.

1. Tamizaje de la mujer embarazada, en la primera consulta prenatal, con un ELISA-VIH.

2. Terapia antirretroviral (TARV) para la mujer embarazada VIH+.

3. Recomendación de vía de parto para la mujer embarazada VIH+.

4. Consejería para la madre $\mathrm{VIH}+$ sobre adherencia al tratamiento, lactancia materna, sexo seguro y nuevos embarazos.

5. Atención de la madre en la Clínica VIH /SIDA de adultos (CVIHa) correspondiente.

6. Atención en el periodo neonatal del hijo (a) de madre $\mathrm{VIH}+$ en el lugar del parto.

7. Atención del hijo (a) de madre VIH+ en la Clínica de Infección por VIH del Hospital Nacional de Niños "Dr. Carlos Sáenz Herrera” (CVIH-HNN). puede ser en cualquier momento que se inicie el control prenatal.

2. Disponibilidad de los medicamentos antirretrovirales, en las presentaciones oral (tabletas y jarabe) e intravenosa que se indican en estas guías. El personal de salud encargado de ofrecer estos medicamentos debe conocer su dosificación y utilización, efectos adversos, contraindicaciones y los efectos reportados sobre el feto y el recién nacido, para ofrecer la consejería adecuada a la madre $\mathrm{VIH}+$.

3. Educación continua del personal de salud a todos los niveles de atención para que la estrategia se desarrolle sin interrupciones.

\section{Actividades para la prevención perinatal del VIH}

La estrategia de prevención debe implementar todas la actividades que se listan en el cuadro 1.

\section{Tamizaje de la mujer embarazada}

Todas las mujeres que acuden a la primera consulta prenatal, deben recibir consejería sobre los beneficios del tamizaje prenatal para VIH. Después de obtener el consentimiento de la mujer para que se realice la prueba de ELISA-VIH, obtener $5 \mathrm{cc}$ de sangre total por punción de una vena periférica, depositarlos en un tubo sin anticoagulante, separar el suero y enviarlo con una orden para "ELISAVIH". Esta orden debe rotularse como "embarazada" y ponerse algún teléfono directo para el reporte inmediato de pruebas positivas.

Las normas internacionales (OPS/OMS) y nacionales (ley general del sida) vigentes, no obligan a que el consentimiento sea por escrito o firmado por la paciente. La recomendación que prevalece es la de simplificar el procedimiento tanto como sea posible.

Los casos con ELISA-VIH reactivo (con cualquier nivel de lectura, los reportes indeterminado o en zona gris se deben considerar como reactivos) deben ser reportados de inmediato por parte del laboratorio al personal de salud que atendió la consulta prenatal. A este nivel no se requiere tomar más pruebas en relación con el diagnóstico de VIH. Con la información del ELISA-VIH la madre debe ser localizada y citada a la mayor brevedad posible en el nivel local, en dicha cita se le debe explicar el resultado y referirla de inmediato a la CVIHa en el hospital que le corresponda según el área de atracción: Calderón Guardia, México, Monseñor Sanabria, Alajuela,o San Juan de Dios. Es conveniente notificar el caso por teléfono al personal de la CVIHa.

A nivel de la CVIHa se solicitará un segundo ELISAVIH y la prueba de Western-Blot. Esta segunda muestra se obtiene igual que la primera; en la orden indicar "ELISAVIH muestra \#2", si es reactiva enviar el suero para "WesternBlot". El resultado de esta segunda muestra debe ser reportado lo antes posible a la CVIHa y la paciente debe ser 
citada para consejería, discusión de los resultados y para ofrecerle el tratamiento adecuado a su condición de mujer embarazada $\mathrm{VIH}+$.

En el caso que la segunda muestra de ELISA-VIH sea reactiva, la CVIHa referirá a la paciente al Servicio de Obstetricia correspondiente para el manejo y seguimiento. No es necesario que el servicio de Obstetricia solicite nuevos estudios en relación con la infección por VIH.

Si la paciente rehúsa la realización del ELISA-VIH durante el embarazo, el personal de salud puede y debe realizarlo al neonato, para cumplir la norma nacional. Sin la mujer no tuvo control prenatal o no se tamizó durante el embarazo, se debe realizar una prueba rápida para VIH (PRVIH) al momento del parto (ver punto III.4). A toda mujer que se presente a la maternidad en labor sin diagnóstico o que desconozca su resultado de la prueba de ELISA-VIH, se le debe realizar una PR-VIH.

\section{Terapia antirretroviral (TARV) de la mujer embarazada VIH +}

La embarazada $\mathrm{VIH}+$ será candidata a recibir durante el embarazo TARV triasociada.

La CVIHa será la encargada de explicar a la madre los resultados de sus estudios de laboratorio, el tratamiento con antirretrovirales que se propone y sus beneficios, efectos adversos, contraindicaciones y las ventajas y desventajas para el recién nacido. Se debe aprovechar para discutir con la madre todos los elementos que componen la estrategia de prevención para la transmisión perinatal del VIH. La madre informada adecuadamente debe dar su consentimiento para iniciar o continuar con el uso de antirretrovirales durante el embarazo.

Las mujeres embarazadas $\mathrm{VIH}+$ referidas o en control en las CVIHa pueden presentar, dependiendo del momento dereferenciay de su experiencia con el uso de antirretrovirales, las siguientes situaciones:

\section{Mujer embarazada infectada por VIH, con 12 o más semanas de gestación y sin TARV previa.}

a. Discutir e iniciar a partir de la semana 12 de gestación, Zidovudina, Lamivudina y Lopinavir/Ritonavir (cuadro 2).

b. Zidovudina iv durante labor de parto (cuadro 2).

c. Notificar al neonatólogo o al pediatra para que discuta con la madre las indicaciones de prevención para el recién nacido.

\section{Mujeres VIH+ con TARV que se inició antes del embarazo.}

Explicar a la madre la TARV que se propone (durante el embarazo, parto y periodo neonatal) y sus beneficios, efectos adversos, contraindicaciones y las ventajas y desventajas para el recién nacido.

\section{Cuadro 2. Dosificación de la TARV en la mujer embarazada}

Terapia ARV durante el embarazo

1. Zidovudina (AZT, Retrovir®)

a. Presentación: cápsulas de $100 \mathrm{mg}$ o tabletas de $300 \mathrm{mg}$

b. Dosificación: $300 \mathrm{mg} \mathrm{c} / 12$ horas vo a partir de la semana 12 o al momento de su detección y durante todo el embarazo.

2. Lamivudina (3TC, Epivir®)

a. Presentación: comprimidos $150 \mathrm{mg}$.

b. Dosificación: $150 \mathrm{mg} \mathrm{c} / 12$ horas.

3. Lopenavir/Ritonavir (LPV, Kaletra®)

a. Presentación: cápsulas 133/33 mg

b. Dosificación: 3 cápsulas c/12 horas.

Terapia ARV durante el parto

4. Zidovudina intravenosa (AZT, Retrovir®)

a. Presentación: frascos de $20 \mathrm{ml}$ con $200 \mathrm{mg}$, es decir $10 \mathrm{mg}$ por $\mathrm{ml}$

b. Dosificación:

i. Dosis de carga: $2 \mathrm{mg} / \mathrm{Kg}$ de peso corporal iv a pasar en la primera hora

ii. Dosis de mantenimiento: de $1 \mathrm{mg} / \mathrm{Kg}$ de peso corporal iv por hora hasta el nacimiento independientemente de la duración de la labor de parto.

c. Preparación: diluir 1 frasco de AZT iv $(200 \mathrm{mg}$ en $20 \mathrm{ml}$ ) en solución de Dextrosa al 5\% o solución Salina, la concentración no debe exceder $4 \mathrm{mg}$ por $\mathrm{ml}$. Se recomienda diluir dos frascos en 360 cc para producir una solución de $1 \mathrm{mg} / \mathrm{cc}$. La solución preparada es estable 8 horas a temperatura ambiente o 24 horas en refrigeración.

d. En caso de cesárea programada, iniciar la Zidovudina intravenosa con las indicaciones anteriores, 3 horas antes del inicio de la cirugía y luego mantener la infusión hasta que se complete el nacimiento.

e. Si no hay Zidovudina intravenosa disponible:

i. Se debe mantener la Zidovudina por vía oral 300 mg (3 cápsulas) cada 3 horas durante el periodo de labor y hasta el nacimiento, administrando la última dosis 3 horas antes de la cesárea. Se puede utilizar el AZT que la madre ha estado tomando durante el embarazo.

ii. Se puede asociar 3TC oral a dosis de 150 $\mathrm{mg} \mathrm{c} / 12$ horas. 
a. Si el diagnóstico del embarazo se hace antes de la semana 12 , discutir con la madre la suspensión temporal de la TARV hasta la semana 12 de embarazo, esto para evitar subdosificaciones de los medicamentos debida a la hiperémesis gravídica que pueden generar resistencia a los medicamentos y para evitar dar la TARV durante el período de mayor riesgo de teratogenicidad.

b. Continuar con la TARV que la mujer esta recibiendo.

c. Si el esquema no incluye Zidovudina, se debe modificar el mismo para que reciba Zidovudina como parte de su TARV.

d. Si está recibiendo Efavirenz, se le debe suspender lo antes posible, modificar el esquema de TARV y discutir con la madre el riesgo para el feto por la exposición a Efavirenz.

e. Implementar las indicaciones para labor y para el recién nacido.

3. Mujeres VIH+, en el periodo de labor, sin TARV previa.

a. Implementar las indicaciones para labor y para el recién nacido.

b. Si en el hospital donde se está atendiendo el parto no hay disponible AZT iv, se puede sustituir por el siguiente esquema: AZT oral $300 \mathrm{mg} \mathrm{c} / 3$ horas durante la labor más lamivudina (3TC) $150 \mathrm{mg}$ una dosis oral c/12 horas durante la labor.

4. Mujeres VIH+, que rehúsan el uso de TARV durante el embarazo.

a. Se les debe dar la consejería adecuada e insistir sobre los beneficios para el recién nacido de la TARV. Si persiste en su decisión, considerar hospitalización e intervención del Patronato Nacional de la Infancia (PANI).

b. Implementar las indicaciones para labor y para el recién nacido.

\section{Vía de parto}

Existe amplia evidencia médica, que demuestra que la cesárea electiva (antes del inicio de labor y de la ruptura de membranas) disminuye por si sola el riesgo de transmisión perinatal del VIH. También se ha demostrado que mientras más instrumentado y prolongado sea el parto vaginal, mayor es el riesgo de transmisión perinatal del VIH. Por lo tanto las recomendaciones en cuanto a la vía de parto son:

1. Mujeres VIH+ en TARV recibiendo TARV triple: parto por cesárea programada a la semana 38 de gestación. Implementar las indicaciones II.1b y II.1c y VI.

2. Mujeres VIH+ en las cuales está indicado un parto por cesárea programada que no es posible realizar: dar vía de parto por vía vaginal, con el mínimo de trauma posible, no instrumentado y sin ruptura artificial de membranas. Implementar las indicaciones II.1b y II.1c y VI.

3. Mujeres VIH+ que inician labor de parto con o sin ruptura de membranas: una vez que la mujer ya inició la labor de parto, lo más importante es que el niño nazca lo más pronto posible, por lo que se debe escoger la vía de parto que logre esto más rápidamente. La prioridad es mayor si hay ruptura de membranas. Siempre que se escoja la vía vaginal debe ser lo más rápida y menos instrumentada posible.

4. Mujeres en prelabor o labor, con condición serológica para VIH desconocida: realizar prueba rápida para VIH y comunicar el resultado de inmediato a los médicos tratantes.

Si la prueba rápida es positiva, tomar de inmediato muestra de sangre para ELISA-VIH y Western-Blot VIH y en tubo con EDTA una muestra para carga viral. Dar vía de parto por cesárea como una emergencia e indicar AZT iv.

El recién nacido debe recibir AZT vo, la lactancia materna se debe suspender y no se debe aplicar la vacuna BCG.

\section{Consejería para la madre VIH+ sobre adherencia al tratamiento, lactancia materna, sexo seguro y nuevos embarazos.}

En el ámbito de la CVIHa y de la consulta prenatal, se debe iniciar un proceso de consejería con la mujer embarazada y su pareja sobre las posibilidades de transmisión del VIH de la madre al niño, la importancia de la adherencia al TARV y de evitar la lactancia materna, planificación de futuros embarazos, posibilidades de esterilización y prácticas de sexo seguro. Dado que en la actualidad la madre puede recibir TARV altamente efectiva que baje su carga viral hasta niveles no detectables, no se debe considerar la infección por VIH como una indicación 'absoluta' de esterilización.

\section{Seguimiento de la madre VIH+ en la CVIHa}

En el ámbito de la CVIHa se tomaran las disposiciones necesarias para el seguimiento y tratamiento de la madre después del parto.

\section{Atención en el periodo neonatal del hijo (a) de madre VIH+}

1. Profilaxis con Zidovudina: el recién nacido debe iniciar tratamiento profiláctico con Zidovudina a las 8 horas de edad y debe mantenerse durante 6 semanas. La Zidovudina se debe dar sin mezclarla con alimentos, a una dosis de $2 \mathrm{mg} / \mathrm{Kg}$ de peso corporal /dosis cada 6 horas. Se debe utilizar la presentación de jarabe, que contiene $10 \mathrm{mg} / \mathrm{ml}$ en frascos de $100 \mathrm{ml}$.

2. Suspensión de la lactancia materna: se le debe explicar a la madre que existe evidencia de que el virus VIH se 
puede transmitir a través de la leche materna y que este factor de riesgo se debe evitar suspendiendo la lactancia materna. Se le debe indicar a la madre que contará con el suministro mensual de una fórmula a base de leche de vaca.

3. Suspensión de la vacunación con BCG: informar a la madre que esta vacuna está compuesta por una bacteria viva, atenuada y explicarle que puede darse el riesgo de diseminación si su hijo presenta defectos en la función de su sistema inmune, como consecuencia de la infección por VIH.

4. Referencia: reportar el caso para seguimiento y para obtener la cita de control, al servicio de Inmunología del Hospital Nacional de Niños “Dr. Carlos Sáenz Herrera, en forma personal o al teléfono 22235125 o 22220122 extensión 4572 o 3309. La hora más adecuada para comunicarse es de 1-3 pm de lunes a viernes.

\section{Atención del niño(a) en la CVIH-HNN}

Durante el período en que se define si el producto de una madre VIH+ está infectado o no, el niño (a) debe recibir durante los primeros 18 meses de edad la atención a los aspectos de crecimiento, desarrollo psicomotor y vacunación en la CVIH-HNN.

En la vacunación, se evita el uso de vacunas con virus o bacterias vivas. No se utiliza BCG y se sustituye la vacuna de Polio oral, por una vacuna de Polio parenteral con base en virus muertos, esto evita también el riesgo de polio asociado a la vacunación en otros miembros de la familia con un defecto en el sistema inmune.

La profilaxis con Trimetroprin/sulfametozaxol evita que la pneumonitis interticial por Pneumocystis jirovecci sea la patología de inicio en los casos de niños (as) que se han infectado por VIH.

Se debe procurar el diagnóstico temprano de infección por $\mathrm{VIH}+$, con la determinación de PCR-ADN en las células mononucleares del niño (a), lo cual permite suspender medicamentos innecesarios cuando no hay infección o iniciar el TARV en los casos en los que se demuestre la transmisión del VIH de la madre al niño (a).

En los pacientes identificados como serovertores, es decir no infectados por VIH, se continúa su control anual por 5 años.

Los pacientes infectados por VIH continúan su tratamiento en la consulta correspondiente de la CVIHHNN.

\section{Referencias}

1. Perinatal HIV Guidelines Working Group. Public Health Service Task Force Recommendations for Use of Antiretroviral Drugs in Pregnant HIV-Infected Women for Maternal Health and Interventions to Reduce Perinatal HIV Transmission in the United States. 8 de Julio. En http:// aidsinfo.nih.gov/ContentFiles/PerinatalGL.pdf. Consultado el 28 de Julio, 2008. 2008 1-98.

2. Connor EN, Sperkling RS, Gelber R et al. Reduction of maternalinfant transmission of human immunodeficiency virus type I with zidovudine treatment. N Engl J Med 1994, 331:1173-1180.

3. The european mode of delivery collaboration. Elective cesarean section versus vaginal delivery in prevention of vertical HIV-1 transmission: a randomized clinical trial. Lancet 1999, 353:10351039.

4. The international perinatal HIV group. The mode of delivery and the risk of vertical transmission of HIV-1- a meta-analysis of 15 prospective cohort studies. N Engl J Med 1999; 340:977-987.

5. American Academy of Pediatrics. Evaluation and treatment of the Human Immunodeficiency Virus-1 exposed infant. Pediatrics 2004; 114:497-505.

6. Read JS, Newell ML. Efficacy and safety of cesarean delivery for prevention of mother-to-child transmission of HIV-1 (Review). Cochrane Database Syst Rev. 2005;(4):CD005479.

7. Inter-agency task team on prevention of HIV infection in pregnant women, mothers and their ghildren. Guidance on global scale-up of the prevention of mother-to-child transmission of HIV. Towards universal access for women, infants and young children and eliminating HIV and AIDS among children. En http://www.who.int/hiv/pub/ guidelines/pmtct scaleup2007/en/print.html . Consultado el 28 de Julio, 2008. 2007 1-38.

6. World Health Organization. HIV/AIDS Programme. Antiretroviral drugs for treating pregnant women and preventing HIV infection in infants: towards universal access. Recommendations for a public health approach. En http://www.who.int/hiv/pub/guidelines/ pmtctguidelines3.pdf . Consultado el 28 de Julio, 2008. 2006 1-92.

7. de Ruiter A, Taylor G, Mercey D, Anderson J, Chakraborty R, Clayden P, et al. British HIV Association and Children's HIV Association guidelines for the management of HIV infection in pregnant women 2008. En http://www.bhiva.org/files/file1030945.pdf . Consultado el 28 de Julio, 2008. 2008 1-113.

8. Burdge DR, Money DM, Forbes JC, Samson LM, Steben M. Canadian consensus guidelines for the care of HIV-positive pregnant women: putting recommendations into practice. CMAJ 2003; 168:1683-1688.

9. Paintsil E, Andiman WA. Care and management of the infant of the HIV-1 infected mother. Semin Perinatol 2007; 31:112-123.

10. Alvarez AM. Rathore MH. Hot topics in pediatric HIV/AIDS. Pediatr Ann 2007; 36:423-432.

11. Warszawski J, Tubiana R, Le Chenadec J, Blanche S, Teglas JP, Dollfus C, et al. Mother-to-child HIV transmission despite antiretroviral therapy in the ANRS French Perinatal Cohort. AIDS 2008; 22: 289-299. 